Corps et culture

Numéro 3 | 1998

Sport et lien social

\title{
Ancrages mythologiques des cultures physiques et projets transgressifs
}

\section{Nancy Midol}

\section{OpenEdition}

1 Journals

\section{Édition électronique}

URL : http://journals.openedition.org/corpsetculture/422

DOI : 10.4000/corpsetculture.422

ISSN : $1777-5337$

\section{Éditeur}

Association Corps et Culture

\section{Édition imprimée}

Date de publication : 1 juin 1998

ISSN : 1268-5631

\section{Référence électronique}

Nancy Midol, «Ancrages mythologiques des cultures physiques et projets transgressifs », Corps et culture [En ligne], Numéro 3 | 1998, mis en ligne le 24 septembre 2007, consulté le 08 septembre 2020. URL : http://journals.openedition.org/corpsetculture/422 ; DOI : https://doi.org/10.4000/ corpsetculture.422

Ce document a été généré automatiquement le 8 septembre 2020

(c) tous droits réservés 


\title{
Ancrages mythologiques des cultures physiques et projets transgressifs
}

\author{
Nancy Midol
}

L'ancrage mythologique de nos cultures corporelles

1 Quels rapports entretiennent les techniques sportives - comme espace de symbolisation de l'intime - avec la mythologie qui fonde l'Occident?

2 L'idéal sportif: Aitius, Citius, Fortius a-t-il un rapport avec l'idéal de la mythologie chrétienne ? Celle-ci disposerait-elle l'occidental à vouloir se dépasser, ou dépasser ses limites?

Le culte de la performance: pour quel dieu?

3 Car il faut questionner pourquoi, sous l'emprise généralisée du culte de la performance (Ehrenberg A., 1991), nos sociétés produisent à travers le Sport, du record pour du record : que cherchent-elles derrière cette quête effrénée, qui ne modifie en rien la vie quotidienne, n'améliore pas la qualité de la vie, ni ne renouvelle la pensée, et se révèle finalement parfaitement déraisonnable, si l'on considère le surentraînement, le dopage, les blessures, la violence sur les stades, la répartition inégale des profits etc.

4 Comment interpréter cette tension vers le dépassement de soi ? N'est-ce pas une sorte de don de soi attendu par une société toute entière 7 Les sportifs de haut niveau y consacrent leur vie, la sacrifient le plus souvent, en font le don. Mais dans ce cas, que représente le Sport, dont plusieurs auteurs (Elias N., 1976, Jeu B., 1977, Bromberger C., 1995, etc.) ont relevé des faits montrant son caractère religieux. Certes, il y a du sacré dans le sport mais à quelle terreur ce sacré fait-il référence? L'a-t-on oubliée? Que nous enseigne la Bible?

« Dieu créa l'homme à son image, à l'image de Dieu, il le créa, homme et femme, il

les créa. » (La sainte Bible, École Biblique de Jérusalem 1955, p. 5)

5 C'est l'idée d'image qui est primordiale ici. Non que l'image soit la reproduction de la représentation physique de Dieu lui-même, mais l'imagination que Dieu projette en 
l'incarnant dans un corps pour permettre la vie terrienne. Cette imagination fonde la dimension sacrée de l'Occident ${ }^{1}$, et sa problématique existentielle.

Construire dieu en nous

Cette image est directionnelle : elle descend de Dieu vers l'humain. Mais, ironie du sens (double sens), il faut qu'elle remonte vers Dieu, il faut que l'humain, dans un mouvement de sublimation radicale, imagine Dieu. C'est pourquoi cette image (ou imagination), jamais acquise est en perpétuel devenir, et, comme Sisyphe, l'occidental s'est condamné à construire et reconstruire son identité, toujours insuffisante.

7 «Dieu a fait l'Homme à son image» est l'assertion mythologique la plus mégalomaniaque qui existe, et la plus narcissique aussi, et qui n'en finit pas d'interpeller l'identité de chacun d'entre nous. Construire Dieu en nous appelle aux actions transcendantes, au dépassement de soi, à inventer les preuves du sublime.

Déjà s'esquissait cette solution chez Platon : « Dieu fut le nom confié à la raison, au logos interrogeant le muthos sous forme de question au-delà de laquelle il n'en est point qui puisse encore ressurgir comme digne du questionnement $»^{2}$.

Donc, pas plus que nous ne pouvons ériger des totems animaliers, nous ne sommes fils ou fille du vent, de l'eau, du feu ou de la terre. Nous sommes d'une autre essence que la planète et le cosmos. On sait, depuis Descartes que ce n'est plus à partir du monde que l'on remonte vers Dieu, mais à partir du moi pensant. Comme le dit plus tard Husserl "nous sommes de race divine", et cela nous permet d'accéder à la raison éthicopratique qui se donne elle-même une loi ${ }^{3}$.

S'identifier en son reflet

10 L'Homme occidental doit donc perpétuellement construire l'image de Dieu pour exister en son reflet. Lacan, se serait-il plus intéressé aux mythologies, aurait-il doublement donné au stade du miroir le rôle fondateur du lien social : de notre lien social. Dans le stade du miroir, l'enfant adhère à la mythologie de ses parents. Ceux-ci, comme Lacan, reconnaissent le geste civilisateur d'appartenance. Il est des nôtres, il s'identifie dans son reflet (sage comme une image viendra plus tard, sur les bancs de l'école, où on aura évacué Dieu, mais pas le projet, celui de se transcender pour se donner à être...).

Faire l'ange pour évacuer la bête

11 Etre la manifestation de Dieu en son image, c'est se mettre dans l'opposition de ceux que Dieu n'a pas élus. Nous ne sommes pas des animaux, d'où l'éducation séculaire du redressement tant physique que moral (Vigarello G., 1978), et sa visibilité esthétique par la danse académique (Legendre P., 1978; Midol N., 1995) etc. Il est intéressant de souligner qu'en chacun de ces projets qui vise à valoriser le trait vertical, les pédagogies ont été d'une sauvagerie terrifiante, en rapport avec la terreur qu'il fallait tenir à distance. Rappelons l'emmaillotage des nouveaux nés, accolés sur une planche et pendus à un clou, ou les longues heures, le corps redressé et engourdi, ou encore les exercices à la barre des danseurs classiques érigés sur leur axe vertébral, droit comme un $i$, travail musculaire et articulaire qui finit par contrefaire la nature en inversant les courbures vertébrales.

Se donner à être dans l'apparence n'est pas nouveau, simplement, dans nos sociétés du spectacle, l'apparence est devenue cette exposition de sur-face qui justifie les programmes de body building, gymnastiques d'entretien, chirurgies esthétiques etc. Les sociétés ont les névroses qu'elles peuvent

Exister sur le modèle distinctif 

la structuration hiérarchisée de nos sociétés avec l'idéal du surpassement, enseigné dès l'école grâce à son organisation sélective, dispositif que l'on reproduit dans le sport et toute autre expression sociale. La distinction acquiert un caractère édifiant (ou édificateur) dans nos sociétés, c'est pourquoi elle justifie d'une mise en scène. Convoquer Dieu en soi, dans l'ordalie, fut l'enjeu des preux chevaliers, en suggérer la présence revient à l'art de la danse ; et on peut se demander si l'enjeu n'est pas encore le même dans la transformation en spectacle, des jeux, des fêtes, des carnavals, et autres manifestations populaires. Le plaisir ne s'échange plus au corps à corps, dans l'émotion du mouvement et de la rencontre, il est médié par un dispositif social qui met en scène, (à distance et en représentation) obligeant d'ailleurs les spectateurs à payer pour assister au spectacle, pendant que d'autres (acteurs, danseurs, sportifs...) sont rétribués pour exhiber une magie de la transcendance.

L'auto-référence

14 Ainsi, coupés des animaux et de la nature, il nous reste la référence à Dieu, c'est-à-dire à nous-mêmes, nous conduisant insensiblement à l'auto-référence, qui s'accompagne d'une forme de dépression chronique, car les autres manquent douloureusement. nature, leurs danses sauvages et leurs rites magiques ${ }^{4}$.

Quant à nous, nos ébats édifiants se déroulent dans des lieux normalisés et homologués, contrefaisant la nature avec des matériaux industriels. La pelouse sur un stade, la haie de lauriers sur un parcours hippique, la vague à Bercy pourraient-ils nous pousser à la rêverie romantique qui, contre le Dieu des Hommes, donnait une âme aux choses inanimées?

17 L'auto-référence se rejoue inlassablement dans la philosophie comme dans le sport où l'on est condamné à la performance.

18 Condamné à inventer notre modèle identificatoire: faire fonctionner une image pour fonctionner nous-mêmes. D'où la fascination séculaire pour l'automate.

L'univers machinique

19 Deus ex machina, comme au théâtre, ce modèle identificatoire fut trouvé dans le modèle de la machine : idéal machinique dit Marie Joseph Biache (1984). Le rapport de l'Homme et de la machine reproduit d'ailleurs celui de Dieu et de l'Homme, ils s'identifient l'un dans l'autre.

20 Ainsi nos techniques du corps, qui donnent sens au geste, ont toujours trouvé dans la machine le modèle de leur fonctionnement. Rappelons la manière dont Marcel Mauss (1950), non sans malice, avouait prendre de l'eau dans la bouche et la recracher pour aider la propulsion de sa nage, s'assimilant à une sorte de bateau à vapeur. Machine mécanique aux rouages des horloges, machine à vapeur au modèle thermodynamique, machine informatique au modèle cybernétique : les modélisation se complexifient, Dieu gagne en intelligence abstraite, et en virtualité.

21 Mais ce n'est évidemment pas seulement le corps qui s'identifie à la machine, la pensée aussi, comme le montre Michel Serres (1977), qui analyse la pensée moderne par l'usage qu'elle fait des principes thermodynamiques pour penser l'histoire (Marx), la situation philosophique (Nietzsche) et le psychisme (Freud). Serres montre comment les systèmes théoriques de la Physique se sont imposés dans la pensée savante et plus tard 
dans la pensée populaire, comme paradigmes pour appréhender l'être humain et ses techniques (Serres M., 1977).

Derniers effets de la mythologie occidentale

L'intrication de l'artifice dans la nature et du technique dans le corps a évolué de façon telle, qu'un seuil est dépassé. La question du lien social fait alors référence à la problématique de l'individu et de l'indifférencié. Pour plusieurs raisons, l'occidental a perdu son statut de sujet pensant, y compris capable d'inventer Dieu. Ce troisième monde, (Beaune J.C., 1991) celui de la technologie avance tout seul, après avoir balayé la suprématie de la pensée philosophique. D'ailleurs il faut prendre particulièrement au sérieux le monde de la technologie managériale qui ne raisonne qu'en termes de produit, de profit, de marché. Ainsi, de nombreux exemples nous montrent que le statut du sujet est équivalent au statut du produit: le travailleur est traité comme un produit, le consommateur aussi: ils sont fabriqués, normalisés, réglementés et manipulés. La science managériale se charge de les faire fonctionner dans l'économie de marché, peut-être comme des automates.

$\mathrm{La}$ " personne patrimoniale »

Les piles cardiaques paraissent archaïques face aux greffes d'organes, ou aux programmes génétiques, etc., l'être humain est devenu un produit composite, sorte de bric-à-brac étonnant, incorporant machines, substances, programmes et codes. Kristeva note que «la personne humaine tend à disparaître comme personne du droit, puisqu'elle est négociée en tant que possédant des organes monnayables. Nous quittons l'ère du sujet pour entrer dans celle de la personne patrimoniale » (1996, p. 18).

Notons aussi que les frontières entre espèces deviennent poreuses. Les maladies actuelles, de la vache folle, de la grippe des poulets et autres manifestations transgéniques inaugurent une nouvelle manière de gérer le vivant. Dans ce cas, l'humain, fut-il occidental, retourne dans le pot commun animalier.

La chair-assistée

Dernièrement un chercheur en biophysique annonçait que des implants microscopiques musculaires étaient en mesure de décupler la force du muscle et sa puissance. Cette information scientifique se donnait comme une offre commerciale: nul doute qu'elle a dû être entendue. Bonjour les nouvelles performances! De toutes façons ce dispositif parait déjà archaïque devant les programmes génétiques qui sont mis au point actuellement par les

Je est un produit « marchandisé »

L'économie mondiale, en terme de marketing ou de management, traque les désirs intimes et collectifs selon une segmentation savante de la population, passant au crible toutes les variables qui en forment ses singularités (classe sociale, genre, moeurs, aspiration spirituelle, âge, aire culturelle, type de spectateurs, idéaux, modes, etc.). Ainsi, personne n'échappe à sa modélisation dans la population scannerisée au rayon managérial. Chacun rejoint son groupe ciblé pour entrer dans son créneau de consommateurs. Aujourd'hui les multinationales s'intéressent au local, à l'intime, à l'inconscient, à tout ce qui peut étendre leur marché en faisant de leurs produits les porteurs de signes nouvellement désirés : c'est ainsi que Nike accole à ses nouveaux produits des signes relatifs à des particularismes locaux. Une réflexion sur le lien social devrait alimenter les savoirs nécessaires à la firme, surtout s'il en ressort quelques nouvelles symboliques qui pourraient avec profit figurer dans «la philosophie du 
produit», exactement comme le slogan: "Just do it» récupérait le défi des situationnistes et correspondait à la mentalité adolescente.

Le nouvel ordre mondial, «normalisateur ", « falsificateur » et falsifié

L'ouvrage "Les fils de Mc Do", de Paul Ariès (1997), met en évidence une sorte de normalisation mondiale du lien social qui fonctionne sur l'assimilation de chacun à l'identité d'un produit. S'élabore une culture mondiale, en deçà des symboliques culturelles qui forment les particularismes, grâce à un matraquage publicitaire sans précédent $^{5}$. Le médium est verbal et visuel. La manipulation joue sur plusieurs tableaux : d'abord sur un mode régressif, Mc Do fait référence à la peur, en certifiant en contrepoint qu'aucun microbe ne vient altérer le produit Mc Do. L'entreprise se présente comme un laboratoire scientifique, élaborant de la haute technologie et produisant la sécurité alimentaire. Ensuite le produit vient là donner du lien social manquant : pour certains Mc Do se présente comme une seconde famille où les enfants fêtent leur anniversaire ; dans les quartiers déshérités, il devient la maison pour tous ; dans les quartiers huppés, le lieu de rencontre des golden boys. Bref, «le hamburger fonctionne comme un pur signifiant, c'est-à-dire comme un simple signe manipulé par des experts en communication" (Ariès P., 1997, p.64). Mc Do contribue ainsi' a segmenter ou encore ethniciser la population, tout à fait consentante et d'ailleurs rassurée: "le touriste occidental pourra désormais parcourir la planète l'esprit tranquille, car il mangera partout le même produit... » (1997, p.15). Seul, le prix du hamburger change, selon que la population environnante est riche ou pauvre.

Tous ensemble, dépassons nos limites

28 Ce modèle managérial fonctionne aussi pour des techniques de culture physique qui nous viennent des USA. Notamment l'aérobie vendue par Jane Fonda, ou encore le Step qui se présentent comme des technologies normalisées qui transforment le sujet en un produit performant. L'animateur de Step, micro incorporé dans son casque, met en marche des centaines d'automates, robotisés par un commandement stéréotypé, sécurisés par l'effet de foule, gesticulant en cadence sur le rythme d'une musique techno. La vision d'une salle de step s'apparente à celle d'une salle de machines industrielles en plein rendement. Mais, contrairement aux critiques de la taylorisation ou du stakhanovisme, chaque machine semble ;jouir dans une sorte de transe, tendue vers le gain d'une amélioration de sa performance : vertige encadré du dépassement de sa limite, sentiment béat d'adhérer à des comportements de gagneurs dans une société qui avance, de participer à la mutation vers le surhumain !

La vogue de la modélisation, y compris dans le champ de l'éducation physique, c'est-àdire du mouvement (movere, émouvoir) reproduit cette logique qui fait passer le sujet au statut de produit. Manquant d'esprit critique, certains robots semblent satisfaits de leur sort.

Dans cette aventure occidentale, qui questionne vigoureusement le lien social, y a-t-il seulement place pour l'expression d'une révolte ? Julia Kristeva en doute face à la force de la normalisation du nouvel ordre mondial, se pose la question du statut du pouvoir et celui de la personne (1996, p. 15).

31 Mais on aurait tort de parler de l'Occident, comme si cette logique occidentale couvrait la planète entière. Si une forme de mondialisation semble avoir généralisé une logique robotisante, elle a fait émerger aussi des conduites marginales, variées et/ou déviantes, qui offrent un contrepoint et font parfois de la résistance.

Les métissages des mythologies, et les logiques identitaires 
32 La culture occidentale est vraiment éclatée, confuse, flottante, diversifiée dans la mesure où de nombreux mouvements se sont ouverts à des métaphysiques exotiques. Dans notre aire culturelle, elles ont imposé des retours à des formes animistes, à travers des danses, des transes, des pratiques thérapeutiques, des croyances, et de nouvelles ou très anciennes pratiques comme le surf notamment. Prenons simplement quels exemples, ça et là, qui suffisent largement à indiquer ces mouvements.

Spiritualités exotiques et techniques du corps

D'un peu partout dans le monde, les spiritualités orientales ont fasciné et parfois converti. Mais c'est un véritable mouvement social qui se déploie, dès les années 40 à partir de la côte pacifique des USA et qui continue sa progression sur la planète entière. Une révolution se fait dans les domaines des arts, des sports, des thérapies corporelles, de la communication, de la diététique, des moeurs, etc. en s'ouvrant aux spiritualités et aux philosophies Bouddhistes, Zen, Taoïstes d'abord, puis dès les années 1970 aux autres spiritualités d'Afrique, d'Amérique, d'Australie, ainsi qu'aux spiritualités ancestrales de son propre territoire (cultes celtes par exemple).

Les initiations aux nouvelles techniques du corps : yoga, méditation, Taï Chi Quan, aïkido..., puis aux rituels de transe, de possession, d'incorporation signifient qu'un ancrage à d'autres fondements mythologiques émerge jusque dans l'aire chrétienne.

Nous reviennent en France, non seulement les rituels druidiques, les pratiques divinatoires des runes, ou des tarots, mais encore des rites amérindiens assortis de leur croyance mythologique, des rites africains, assortis de danses, de combats, de musiques... Les initiations se font en Casamance ou encore au Brésil pour les pratiques du Lumbanda ou du Condemblé, ou encore en Australie pour apprendre à servir la nature. Elles s'implantent aussi en Occident, et l'on peut apprendre la danse africaine, la capoeira, suivre des rituels de possession etc. A ces occasions, les occidentaux pratiquent ce que leur mythologie leur avait interdit : la transe, la fusion de la nature, la recherche d'une filiation totémique, l'expérience d'incorporation d'ancêtres, d'esprits etc. Nous ne parlons pas ici de sectes nouvelles, mais de quêtes mystiques qui s'ouvrent à d'autres traditions, avec ce que cela suppose d'apprentissages autres concernant les techniques corporelles et les liens sociaux.

Les dieux du surf

36 Il en est de même des groupes d'initiés-surfeurs, qui ont adhéré à la mythologie hawaïenne et pratiquent les rituels sur l'eau. Il faut aussi remarquer que cette ouverture à une autre croyance a permis que d'autres groupes, pratiquant d'autres activités sportives comme l'escalade ou la planche à roulettes, s'inventent des rituels initiatiques, assortis d'une nouvelle philosophie de l'existence.

37 C'est comme si, après avoir accédé à l'idée de l'égalité, les occidentaux étaient en mesure de respecter d'autres croyances fondatrices et même d'y adhérer. Pendant que la masse est manipulée, des élites ou des marginaux affirment une démarche identitaire active. Qu'on les appelle Tribus ou Castes, s'élabore là, une autre forme de relation sociale, une autre appréhension de la collectivité, avec ses formes discriminantes et solidaires, ses régressions et ses sublimations.

Le créneau des sectes

38 Dans cette hétérogénéité théorique, affective, identitaire, les personnes flottent entre plusieurs sources mythologiques, plusieurs logiques, plusieurs rationalités. La construction identitaire s'accommode de certaines contradictions. L'éclatement des 
repères et des cadres mentaux donne une certaine disponibilité aux personnes en quête de spiritualité, pour trouver des voies métaphysiques dans la Tradition (bouddhisme, par exemple) ou encore dans des voies nouvelles : temple solaire, église scientiste, secte Moon etc. dont les gourous se conduisent en astucieux managers ayant juteusement ciblé le besoin de spiritualité.

Conclusion

En chacun des ces paragraphes apparait la problématique de la transgression. Du hors limites, de l'extrême, de l'exotisme aux nouvelles sectes, le lien social marque un projet transgressif. Mais la problématique de la transgression n'est pas celle de la révolte. $\mathrm{La}$ transgression fonctionne comme une forme de jouissance, et comme une nouvelle dépendance. Suivre une conduite aux représentations transgressives concerne les plus exigeants comme les gourmands savourant un Kim Cône glacé enrobé de noisettes au chocolat.

Car, à force de réduire la dimension symbolique (qui enchante le monde) à des signes marchands (qui manipulent le monde), l'exploitation économique nous pousse à nous protéger dans des groupes discrets, secrets, ou derrière des formes ésotériques anciennes ou nouvelles.

L'avènement d'un ordre mondial normalisateur génère et règle ces jeux subtils entre le pouvoir (devenu vacant), et les différentes formes de transgressions (Kristeva J., 1996, 17).

Plus que jamais la phrase de Voltaire fait sens : « Pour vivre heureux, vivons cachés !»

Mais comment vivre caché avec ces bandes de chercheurs opiniâtres dont la passion est de traquer l'inconnu, le secret, l'ignorance, les défenses, etc. !

\section{BIBLIOGRAPHIE}

Ariès P. (1997) Les Fils de Mc Do, L'Harmattan, Paris.

Biache M.A. (1 984), Le Monstrueux et le machinique, Anthropologie des Techniques du corps, AFRAPS/ Université de Nice, Nice, 379 -394.

Beaune J.-C. (1991) Raison et déraison technique; communication au colloque Technologie des APS, AFRAPS, Strasbourg.

Bromberger C. (1995) Le match de foot, ethnologie d'une passion partisane à Marseille, Naples, et Turin, Editions de la Maison des Sciences de l'Homme. Paris.

Debord G. (1969) La Société du spectacle, Minuit, Paris.

Ehrenberg A.(1 991) Le Culte de la performance, Paris, Calmann-Lévy.

Elias N. (1979) Le Sport, l'État, la violence, Actes de la recherche en Sciences Sociales, 6, Paris.

Jeu B. (1977) Le Sport, l'émotion, l'espace, Paris, Vigot.

Kristeva J. (1996) Sens et non-sens de la révolte, Fayard, Paris. 
Legendre P (1978) La Passion d'être un autre, Seuil Paris,

Mauss M. (1950) Sociologie et anthropologie, Paris, PUF.

Midol N. (1995) Démiurgie dans les Sports et la Danse, Paris, L'Harmattan.

Serres M. (1977) Hermès IV. La distribution, Minuit, Paris.

Vigarello G. (1978) Le Corps redressé, Paris, Ed. Universitaire.

\section{NOTES}

1. Chez les musulmans et les juifs, il est interdit de représenter cette image. Il existe une terreur autour de cet interdit.

2. Encyclopaedia Universalis, 1995-1996, Dieu - problématique philosophique.

3. Husserl, plus explicite, écrit : Dieu est en moi comme raison éthico-pratique se donnant elle-même la loi, et c'est ce qui fait que nous sommes originairement de « race divine ». Encyclopaedia Universalis, 1995-1996, Dieu - problématique philosophique).

4. D'ailleurs nous ne leur laissons pas, nous les convertissons.

5. Plus de 2 millions de dollars par jour dans la seule publicité télévisée, et 5 milliards de francs annuellement.

\section{RÉSUMÉS}

Quels rapports les techniques sportives entretiennent avec la mythologie chrétienne? Pourquoi le Sport produit-il du record pour du record? Le mythe de Sisyphe pourrait illustrer comment chacun est condamné à toujours reconstruire son identité, sous l'emprise généralisée du culte de la performance. Longtemps identifié aux modèles techniques, les occidentaux aujourd'hui sont traités comme un produit: le travailleur comme le consommateur est fabriqué, normalisé, manipulé. Qu'il participe à une séance d'aérobic ou de Step, il fonctionne comme un objet modélisé. Mais ce nouvel ordre mondial normalisateur génère en contrepoint des jeux subtils de transgressions. Ces jeux sont identifies dans les nouvelles cultures corporelles.

Which reports the sporty techniques maintain with the Christian mythology? The myth of Sisyphus could illustrate how each one is condemned to keep rebuilding one's identity, under the ascendancy generalized of the cult of the performance. Western people are identified as technical models, and today they are treated like products: the worker as the consumer is manufactured, normalized, manipulated. The fact one is participating to an aerobic dance period or a Step training, shows that one function as a modelised object. But this new normalised worldwide order generates in some other ways the subtle transgressions games. These games are identified in the new bodily cultures. 
INDEX

Mots-clés : mondialisation, cultures corporelles, transgression

Keywords : globalization, bodily cultures, mythology

\section{AUTEUR}

NANCY MIDOL

Faculté des Sciences du Sport et de l'Education Physique. Université de Nice, Sophia-Antipolis 